\title{
Common fixed point theorems for non-self mappings of nonlinear contractive maps in convex metric spaces
}

\author{
Kanayo Stella Eke ${ }^{\mathrm{a}, *}$, Bijan Davvaz ${ }^{\mathrm{b}}$, Jimevwo Godwin Oghonyon ${ }^{\mathrm{a}}$ \\ a Department of Mathematics, Covenant University, Canaanland, KM 10 ldiroko Road, P. M. B. 1023, Ota, Ogun State, Nigeria. \\ ${ }^{b}$ Department of Mathematics, Yazd University, Yazd, Iran.
}

\begin{abstract}
In this paper, we introduce a class of nonlinear contractive mappings in metric space. We also establish common fixed point theorems for these pair of non-self mappings satisfying the new contractive conditions in the convex metric space . An example is given to validate our results. The results generalize and extend some results in literature.
\end{abstract}

Keywords: Convex metric space, nonlinear contractive mapping, non-self mapping, common fixed point, coincidentally commuting.

2010 MSC: 47H10.

(C)2018 All rights reserved.

\section{Introduction and preliminary definitions}

The metrically fixed point theorems for contraction self-mappings have find applications in various areas in mathematics and economics. Many authors have proved the existence and uniqueness of common fixed points of contraction self mappings in metric spaces and its generalizations (see Karapinar [12], Abdeljawad et al. [2], Aydi et al. [6], Aydi et al. [7]). Much work have been done on the approximation of fixed points of contraction mappings (see [3, 14, 15]). Kirk [13] extended the metric space to metric space of hyperbolic type by placing Krasnoselskii's result (for $f_{\alpha}=(1-\alpha) I+\alpha I$ for some $\alpha \in(0,1)$ ) in the framework of convex metric space. In convex metric spaces occur cases where the involved function is not necessarily a self-mapping of a closed subset. Assad [4] and Assad and Kirk [5] proved the first fixed point result for multivalued non-self mappings in a metric space $(X, d)$. Many authors have studied the existence and uniqueness of fixed and common fixed point results for non-self contraction mappings, see Ćirić [8], Imdad and Kumar [11], Rhoades [17], Sumitra et al. [18].

Throughout our consideration, we suppose that $(X, d)$ is a metric space which contains a family $L$ of metric segments (isometric images of real line segment) such that

(a) each two points $x, y \in X$ are endpoints of exactly one number $\operatorname{seg}[x, y]$ of $L$, and

(b) if $u, x, y \in X$ and if $z \in \operatorname{seg}[x, y]$ satisfies $d(x, z)=\lambda d(x, y)$ for $\lambda \in[0,1]$, then

$$
d(u, z) \leqslant(1-\lambda) d(u, x)+\lambda d(u, y) .
$$

\footnotetext{
${ }^{*}$ Corresponding author

Email address: kanayo.eke@covenantuniversity.edu.ng (Kanayo Stella Eke)

doi: $10.22436 /$ jmcs.018.02.06
} 
A space of this type is called convex metric space of Takahashi ([19]).

Definition 1.1 ([1]). Let $f$ and $g$ be self -maps of a set $X$. If $w=f x=g x$ for some $x$ in $X$, then $x$ is called a coincidence point of $f$ and $g$, and $w$ is called a point of coincidence of $f$ and $g$. Self-maps $f$ and $g$ are said to be coincidentally commuting if they commute at their coincidence point; i.e., if $f x=g x$ for some $x \in X$, then $f g x=g f x$.

Ćirić et al. [9] proved the following common fixed point theorem in metric space of hyperbolic type.

Theorem 1.2. Let $\mathrm{X}$ be a metric space of hyperbolic type, $\mathrm{K}$ a non-empty closed subset of $\mathrm{X}$, and $\delta \mathrm{K}$ the boundary of $\mathrm{K}$. Let $\delta \mathrm{K}$ be non-empty and let $\mathrm{T}: \mathrm{K} \rightarrow \mathrm{X}$ and $\mathrm{f}: \mathrm{K} \cap \mathrm{T}(\mathrm{K}) \rightarrow \mathrm{X}$ be two non-self-mappings satisfying the following conditions:

$$
d(f x, f y) \leqslant \phi\left(\max \left\{\frac{d(T x, T y)}{2}, d(T x, f x), d(T y, f y), \min \{d(T x, f y), d(f x, T y)\}, \frac{d(T y, f x)+d(T x, f y)}{q}\right\}\right)
$$

for all $x, y \in X$, where $\phi:[0, \infty) \rightarrow[0, \infty)$ is a real function which has the following properties

- $\phi(\mathrm{t})<\mathrm{t}$ for $\mathrm{t}>0$ and $\phi(\mathrm{t})$ is non-decreasing.

Suppose that $\mathrm{F}$ and $\mathrm{T}$ have the following additional properties:

(1) $\delta \mathrm{K} \subset \mathrm{TK}, \mathrm{fK} \cap \mathrm{K} \subset \mathrm{TK}$;

(2) $\mathrm{T} x \in \delta \mathrm{K} \Rightarrow \mathrm{f} x \in \mathrm{K}$;

(3) $\mathrm{K} \cap \mathrm{T}(\mathrm{K})$ is complete.

Then, there exists a coincidence point $z$ in $\mathrm{Z}$. Moreover, if $(\mathrm{f}, \mathrm{T})$ are coincidentally commuting, then $z$ is the unique common fixed point of $\mathrm{f}$ and $\mathrm{T}$.

Radenović and Rhoades [16] proved the analog of Theorem 1.2 in the setting of cone metric space of Huang and Zhang [10] for $\phi(t)=k t$, where $k \in[0,1)$.

The purpose of this paper is to introduce a new pair of non-linear contractive type non-self mappings which satisfy a new contractive condition and prove common fixed point theorems in convex metric space. Our results generalize and extend the results of Radenović and Rhoades [16] and Ćirić et al. [9] and other related results in the literature.

The following section gives the definition of new non-linear contractive type of non-self mappings defined in the metric space. The existence and uniqueness of the common fixed point of these operators in the setting of convex metric space is proved.

\section{Main results}

Definition 2.1. Let $(X, d)$ be metric space, $K$ be a non-empty closed subset of $X$, and $f, T: K \rightarrow X$. If $f$ and $T$ satisfy the condition $d(f x, f y) \leqslant \lambda \cdot u(x, y)$, where

$$
u(x, y) \in\left\{\frac{d(T x, T y)}{2}, d(T x, f x), d(T y, f y), \min \{d(T x, f y), d(T y, f x)\}, \frac{d(T x, f y)+d(T y, f x)}{q}\right\}
$$

for all $x, y \in C, 0<\lambda<1, q \geqslant 2-\lambda$, then, $f$ is called a generalized contractive mapping of $K$ into $X$.

We state and prove our main result as follows.

Theorem 2.2. Let $\mathrm{X}$ be a convex metric space, $\mathrm{K}$ a non-empty closed subset of $\mathrm{X}$, and $\delta \mathrm{K}$ the boundary of $\mathrm{K}$. Let $\delta \mathrm{K}$ be nonempty and let $\mathrm{T}: \mathrm{K} \rightarrow \mathrm{X}$ and $\mathrm{f}: \mathrm{K} \cap \mathrm{T}(\mathrm{K}) \rightarrow \mathrm{X}$ be the generalized contractive mappings of $\mathrm{K}$ into $\mathrm{X}$, and

(1) $\delta \mathrm{K} \subset \mathrm{TK}, \mathrm{fK} \cap \mathrm{K} \subset \mathrm{TK}$;

(2) $\mathrm{T} x \in \delta \mathrm{K} \Rightarrow \mathrm{f} x \in \mathrm{K}$;

(3) $\mathrm{fK} \cap \mathrm{K}$ is complete. 
Then there exists a coincidence point $z$ in $X$. Moreover, if $\mathrm{f}$ and $\mathrm{T}$ are coincidentally commuting, then $z$ is the unique common fixed point of $\mathrm{f}$ and $\mathrm{T}$.

Proof. Let $x \in \delta K$ be arbitrary. We construct three sequences $\left\{x_{n}\right\}$ and $\left\{z_{n}\right\}$ in $K$ and a sequence $\left\{y_{n}\right\}$ in $\mathrm{fK} \subset \mathrm{X}$ as follows. Choose $z_{\mathrm{n}}=\mathrm{x}$. Since $z_{0} \in \delta \mathrm{K}$, it follows that there exists $x_{0} \in \mathrm{K}$ such that $z_{0}=\mathrm{T} x_{0} \in \delta K$. By (3), we have $f x_{0} \in K$. Now, choose $y_{1}=T x_{1}$ with $y \in f K \subset X$. This implies that $f x_{0} \in f K \cap K \subset T K$. Set $y_{1}=f x_{0}$, we choose $x_{1} \in K$ such that $T x_{1}=f x_{0}$. Hence $z_{1}=T x_{1}=f x_{0}=y_{1}$. This gives $\mathrm{y}_{2}=\mathrm{fx}_{1}$.

Since $y_{2} \in f K \cap K$, it follows that $y_{2} \in T K$ by (2). Let $x_{1} \in K$ with $z_{1}=T x_{1} \in \delta K$ such that $z_{2}=T x_{2}=$ $f x_{1}=y_{2}$. If $f x_{1}=y_{2} \notin K$, then there exists $z_{2} \in \delta K\left(z_{2} \notin y_{2}\right)$ such that $z_{2} \in \operatorname{seg}\left[y_{1}, y_{2}\right]$. Since $x_{2} \in K$, then by (i) we have $T x_{2}=z_{2}$. Hence $z_{2} \in \delta K \cap \operatorname{seg}\left[y_{1}, y_{2}\right]$.

We can choose $y_{3} \in f K \cap K$, and by (2), $y_{3} \in T K$. Let $x_{2} \in K$ such that $T x_{3}=y_{3}=f x_{2}$. Continuing in the process, we construct three sequences $\left\{x_{n}\right\} \subseteq K,\left\{z_{n}\right\} \subseteq K$, and $\left\{y_{n}\right\} \subseteq f K \subset X$ such that

(a) $y_{n}=f x_{n-1}$

(b) $z_{\mathrm{n}}=\mathrm{T} x_{\mathrm{n}}$;

(c) $z_{n}=y_{n}$ if and only if $y_{n} \in K$;

(d) $z_{n} \notin y_{n}$ whenever $y_{n} \notin K$ and $z_{n} \in \delta K$ such that

$$
z_{n} \in \delta K \cap \operatorname{seg}\left[f x_{n-2}, f x_{n-1}\right] .
$$

This proves that $f$ and $T$ are non-self mappings.

Remark 2.3. By (d) if $z_{\mathfrak{n}} \neq y_{n}$, then $z_{\mathfrak{n}} \in \delta K$ and combining (b), (2), and (a) we obtain $z_{n+1}=y_{n+1}$. Likewise $z_{n-1}=y_{n-1} \in K$. If $z_{n-1} \in \delta K$, then it implies $z_{n}=y_{n} \in K$.

Next, we show that $x_{n} \neq x_{n+1}$ for all $n$. From (a), (b), (c), and (d) we can establish three possibilities as follows.

(1) $z_{\mathrm{n}}=\mathrm{y}_{\mathrm{n}} \in \mathrm{K}$ and $z_{\mathrm{n}+1}=\mathrm{y}_{\mathrm{n}+1}$;

(2) $z_{n}=y_{n} \in K$ but $z_{n+1} \neq y_{n+1}$;

(3) $z_{n} \neq y_{n} \in K$ in which case $z_{n} \in \delta K \cap \operatorname{seg}\left[f x_{n-2}, f x_{n-1}\right]$.

Now, we consider the following cases.

Case (1): Let $z_{\mathfrak{n}}=y_{n} \in K$ and $z_{n+1}=y_{n+1}$. Using equation (2.1) we obtain

$$
d\left(z_{n}, z_{n+1}\right)=d\left(y_{n}, y_{n+1}\right)=d\left(f x_{n-1}, f x_{n}\right) \leqslant \lambda \cdot u_{n},
$$

where

$$
\begin{aligned}
u_{n} \in\{ & \frac{d\left(T x_{n-1}, T x_{n}\right)}{2}, d\left(T x_{n-1}, f x_{n-1}\right), d\left(T x_{n}, f x_{n}\right), \\
& \left.\min \left\{d\left(T x_{n-1}, f x_{n}\right), d\left(T x_{n}, f x_{n-1}\right)\right\}, \frac{d\left(T x_{n-1}, f x_{n}\right)+d\left(T x_{n}, f x_{n-1}\right)}{q}\right\} \\
= & \left\{\frac{d\left(z_{n-1}, z_{n}\right)}{2}, d\left(z_{n-1}, y_{n}\right), d\left(z_{n}, y_{n+1}\right), \min \left\{d\left(z_{n-1}, y_{n+1}\right), d\left(z_{n}, y_{n}\right)\right\},\right. \\
& \left.\frac{d\left(z_{n-1}, y_{n+1}\right)+d\left(z_{n}, y_{n}\right)}{q}\right\} \\
= & \left\{\frac{d\left(z_{n-1}, z_{n}\right)}{2}, d\left(z_{n-1}, z_{n}\right), d\left(z_{n}, z_{n+1}\right), 0, \frac{d\left(z_{n-1}, z_{n+1}\right)+0}{q}\right\} \\
= & \left\{\frac{d\left(z_{n-1}, z_{n}\right)}{2}, d\left(z_{n-1}, z_{n}\right), d\left(z_{n}, z_{n+1}\right), 0, \frac{d\left(z_{n-1}, z_{n}\right)+d\left(z_{n}, z_{n+1}\right)}{q}\right\} .
\end{aligned}
$$

Obviously, there are infinite many $n$ such that at least one of the following cases holds:

(i) $\mathrm{d}\left(z_{\mathrm{n}}, z_{\mathrm{n}+1}\right) \leqslant \lambda \cdot \frac{\mathrm{d}\left(z_{\mathrm{n}-1}, z_{\mathfrak{n}}\right)}{2} \leqslant \lambda \mathrm{d}\left(z_{\mathrm{n}-1}, z_{\mathfrak{n}}\right)$;

(ii) $\mathrm{d}\left(z_{\mathrm{n}}, z_{\mathrm{n}+1}\right) \leqslant \lambda \cdot \mathrm{d}\left(z_{\mathrm{n}-1}, z_{\mathfrak{n}}\right)$; 
(iii) $\mathrm{d}\left(z_{\mathrm{n}}, z_{\mathrm{n}+1}\right) \leqslant \lambda \cdot \mathrm{d}\left(z_{\mathrm{n}}, z_{\mathrm{n}+1}\right)$ (a contradiction);

(iv)

$$
\begin{aligned}
d\left(z_{n}, z_{n+1}\right) & \left.\leqslant \lambda \cdot \frac{d\left(z_{n-1}, z_{n}\right)+d\left(z_{n}, z_{n+1}\right)}{q}\right\} \leqslant \frac{\lambda}{q}\left(d\left(z_{n-1}, z_{n}\right)+d\left(z_{n}, z_{n+1}\right)\right), \\
\left(1-\frac{\lambda}{q}\right) d\left(z_{n}, z_{n+1}\right) & \leqslant \frac{\lambda}{q} d\left(z_{n-1}, z_{n}\right), \\
d\left(z_{n}, z_{n+1}\right) & \leqslant \frac{\lambda}{q} \times \frac{q}{q-\lambda} \cdot d\left(z_{n-1}, z_{n}\right) \leqslant \frac{\lambda}{q-\lambda} \cdot d\left(z_{n-1}, z_{n}\right) .
\end{aligned}
$$

Combining the four cases (i), (ii), (iii), and (iv) we obtain

$$
d\left(z_{n}, z_{n+1}\right) \leqslant k \cdot d\left(z_{n-1}, z_{n}\right),
$$

where $k=\max \left\{\lambda, \frac{\lambda}{q-\lambda}\right\}$.

Case 2: Let $z_{n}=y_{n} \in K$ but $z_{n+1} \neq y_{n+1}$. Then $z_{n+1} \in \delta K \cap \operatorname{seg}\left[y_{n}, y_{n+1}\right]$. From equation (1.1) with $u=y$, we obtain

$$
d(y, z) \leqslant(1-\lambda) d(x, y)
$$

Therefore, we have

$$
d(x, y) \leqslant d(x, z)+d(z, y) \leqslant \lambda d(x, y)+(1-\lambda) d(x, y)=d(x, y)
$$

Hence,

$$
z \in \operatorname{seg}[x, y] \Rightarrow d(x, z)+d(z, y)=d(x, y),
$$

because $z_{n+1} \in \operatorname{seg}\left[y_{n}, y_{n+1}\right]=\operatorname{seg}\left[z_{n}, y_{n+1}\right]$. We have

$$
d\left(z_{n}, z_{n+1}\right)=d\left(y_{n}, z_{n+1}\right)=d\left(y_{n}, y_{n+1}\right)-d\left(z_{n+1}, y_{n+1}\right)<d\left(y_{n}, y_{n+1}\right) .
$$

From Case (1), we obtain

$$
d\left(y_{n}, y_{n+1}\right) \leqslant \lambda \cdot d\left(z_{n-1}, z_{n}\right) .
$$

Case (3): $z_{n} \neq y_{n}$. Then $z_{n} \in \delta K \cap \operatorname{seg}\left[f x_{n-2}, f x_{n-1}\right]$, i.e., $z_{n} \in \delta K \cap \operatorname{seg}\left[y_{n-1}, y_{n}\right]$. By Remark 2.3 we have $z_{n+1}=y_{n+1}$ and $z_{n-1}=y_{n-1}$. This implies that

$$
\begin{aligned}
\mathrm{d}\left(z_{n}, z_{n+1}\right)=d\left(z_{n}, y_{n+1}\right) & \leqslant d\left(z_{n}, y_{n}\right)+d\left(y_{n}, y_{n+1}\right) \\
& =d\left(z_{n-1}, y_{n}\right)-d\left(z_{n}, z_{n-1}\right)+d\left(y_{n}, y_{n+1}\right) \\
& =d\left(y_{n-1}, y_{n}\right)-d\left(z_{n}, z_{n-1}\right)+d\left(y_{n}, y_{n+1}\right) .
\end{aligned}
$$

We shall find $d\left(y_{n-1}, y_{n}\right)$ and $d\left(y_{n}, y_{n+1}\right)$. Since $z_{n-1}=y_{n-1}$, we can conclude that

$$
d\left(y_{n-1}, y_{n}\right) \leqslant \lambda \cdot d\left(z_{n-2}, z_{n-1}\right),
$$

with respect to Case (2).

Now, we obtain

$$
d\left(y_{n}, y_{n+1}\right)=d\left(f x_{n-1}, f x_{n}\right) \leqslant \lambda \cdot u_{n}
$$

where

$$
\begin{aligned}
u_{n} \in & \left\{\frac{d\left(T x_{n-1}, T x_{n}\right)}{2}, d\left(T x_{n-1}, f x_{n-1}\right), d\left(T x_{n}, f x_{n}\right), \min \left\{d\left(T x_{n-1}, f x_{n}\right), d\left(T x_{n}, f x_{n-1}\right)\right\},\right. \\
& \left.\frac{d\left(T x_{n-1}, f x_{n}\right)+d\left(T x_{n}, f x_{n-1}\right)}{q}\right\} \\
= & \left\{\frac{d\left(z_{n-1}, z_{n}\right)}{2}, d\left(z_{n-1}, y_{n}\right), d\left(z_{n}, y_{n+1}\right), \min \left\{d\left(z_{n-1}, y_{n+1}\right), d\left(z_{n}, y_{n}\right)\right\}, \frac{d\left(z_{n-1}, y_{n+1}\right)+d\left(z_{n}, y_{n}\right)}{q}\right\} \\
= & \left\{\frac{d\left(z_{n-1}, z_{n}\right)}{2}, d\left(z_{n-1}, y_{n}\right), d\left(z_{n}, z_{n+1}\right), d\left(z_{n}, y_{n}\right), \frac{d\left(z_{n-1}, z_{n+1}\right)+d\left(z_{n}, y_{n}\right)}{q}\right\} .
\end{aligned}
$$


(i) $d\left(y_{n}, y_{n+1}\right) \leqslant \lambda \cdot \frac{d\left(z_{n-1}, z_{n}\right)}{2} \leqslant \lambda d\left(z_{n-1}, z_{n}\right)$;

(ii) $d\left(y_{n}, y_{n+1}\right) \leqslant \lambda \cdot d\left(z_{n-1}, y_{n}\right)=\lambda d\left(y_{n-1}, y_{n}\right)$;

(iii) $d\left(y_{n}, y_{n+1}\right) \leqslant \lambda \cdot d\left(z_{n}, z_{n+1}\right)$;

(iv) $d\left(y_{n}, y_{n+1}\right) \leqslant \lambda \cdot d\left(z_{n}, y_{n}\right)=\lambda \cdot\left\{d\left(z_{n}, z_{n-1}\right)-d\left(z_{n-1}, y_{n}\right) \leqslant \lambda \cdot d\left(z_{n}, z_{n-1}\right)\right.$;

(v)

$$
\begin{aligned}
d\left(y_{n}, y_{n+1}\right) & \leqslant \frac{\lambda}{q}\left\{d\left(z_{n-1}, y_{n+1}\right)+d\left(y_{n}, z_{n}\right)\right\} \\
& \leqslant \frac{\lambda}{q}\left(d\left(z_{n-1}, z_{n}\right)+d\left(z_{n}, y_{n+1}\right)+d\left(y_{n-1}, y_{n}\right)-d\left(z_{n-1}, z_{n}\right)\right) \\
& \leqslant \frac{\lambda}{q}\left(d\left(y_{n-1}, y_{n}\right)+d\left(z_{n}, z_{n+1}\right)\right),
\end{aligned}
$$

$z_{n+1}=y_{n+1}, z_{n-1}=y_{n-1}$ and $d\left(y_{n-1}, y_{n}\right) \leqslant \lambda \cdot d\left(z_{n-2}, z_{n-1}\right)$, we obtain

$$
d\left(y_{n}, y_{n+1}\right) \leqslant \frac{\lambda}{q}\left(\lambda \cdot d\left(z_{n-2}, z_{n-1}\right)+d\left(z_{n}, z_{n+1}\right)\right)
$$

Substituting equation (2.3) and the above case in equation (2.2) yields,

(vi)

$$
\begin{aligned}
\mathrm{d}\left(z_{\mathfrak{n}}, z_{\mathrm{n}+1}\right) & \leqslant \lambda \cdot \mathrm{d}\left(z_{\mathfrak{n}-2}, z_{\mathfrak{n}-1}\right)-\mathrm{d}\left(z_{\mathfrak{n}-1}, z_{\mathfrak{n}}\right)+\lambda \cdot \mathrm{d}\left(z_{\mathfrak{n}-1}, z_{\mathfrak{n}}\right) \\
& =\lambda \cdot \mathrm{d}\left(z_{\mathfrak{n}-2}, z_{\mathfrak{n}-1}\right)+\lambda \mathrm{d}\left(z_{\mathfrak{n}-1}, z_{\mathfrak{n}}\right) \\
& \leqslant 2 \lambda \max \left\{\mathrm{d}\left(z_{\mathfrak{n}-2}, z_{\mathfrak{n}-1}\right), \mathrm{d}\left(z_{\mathfrak{n}-1}, z_{\mathfrak{n}}\right)\right\} \leqslant \lambda \mathrm{d}\left(z_{\mathfrak{n}-2}, z_{\mathfrak{n}-1}\right)
\end{aligned}
$$

(vii)

$$
\mathrm{d}\left(z_{\mathfrak{n}}, z_{\mathrm{n}+1}\right) \leqslant \lambda \cdot \mathrm{d}\left(z_{\mathrm{n}-2}, z_{\mathrm{n}-1}\right)-\mathrm{d}\left(z_{\mathrm{n}-1}, z_{\mathfrak{n}}\right)+\lambda \cdot \lambda \mathrm{d}\left(z_{\mathrm{n}-2}, z_{\mathrm{n}-1}\right) \leqslant\left(\lambda+\lambda^{2}\right) \mathrm{d}\left(z_{\mathfrak{n}-2}, z_{\mathfrak{n}-1}\right)
$$

(viii)

$$
\begin{aligned}
\mathrm{d}\left(z_{\mathfrak{n}}, z_{\mathfrak{n}+1}\right) & \leqslant \lambda \cdot \mathrm{d}\left(z_{\mathfrak{n}-2}, z_{\mathfrak{n}-1}\right)-\mathrm{d}\left(z_{\mathfrak{n}-1}, z_{\mathfrak{n}}\right)+\lambda \mathrm{d}\left(z_{\mathfrak{n}}, z_{\mathfrak{n}+1}\right), \\
(1-\lambda) \mathrm{d}\left(z_{\mathfrak{n}}, z_{\mathfrak{n}+1}\right) & \leqslant \lambda \cdot \mathrm{d}\left(z_{\mathrm{n}-2}, z_{\mathfrak{n}-1}\right)-\mathrm{d}\left(z_{\mathfrak{n}-1}, z_{\mathfrak{n}}\right), \\
\mathrm{d}\left(z_{\mathfrak{n}}, z_{\mathfrak{n}+1}\right) & \leqslant \frac{\lambda}{1-\lambda} \mathrm{d}\left(z_{\mathfrak{n}-2}, z_{\mathfrak{n}-1}\right)
\end{aligned}
$$

(ix)

$$
\begin{aligned}
& \mathrm{d}\left(z_{\mathrm{n}}, z_{\mathrm{n}+1}\right) \leqslant \lambda \cdot \mathrm{d}\left(z_{\mathrm{n}-2}, z_{\mathrm{n}-1}\right)-\mathrm{d}\left(z_{\mathrm{n}-1}, z_{\mathrm{n}}\right)+\lambda \mathrm{d}\left(z_{\mathrm{n}}, z_{\mathrm{n}-1}\right) \\
& \leqslant \lambda \cdot \mathrm{d}\left(z_{\mathfrak{n}-2}, z_{\mathfrak{n}-1}\right)+\lambda \mathrm{d}\left(z_{\mathrm{n}}, z_{\mathfrak{n}-1}\right) \\
& \leqslant 2 \lambda \max \left\{\mathrm{d}\left(z_{\mathrm{n}-2}, z_{\mathrm{n}-1}\right), \mathrm{d}\left(z_{\mathfrak{n}}, z_{\mathfrak{n}-1}\right)\right\} \\
& \leqslant \lambda \max \left\{d\left(z_{\mathfrak{n}-2}, z_{\mathfrak{n}-1}\right), \mathrm{d}\left(z_{\mathfrak{n}}, z_{\mathfrak{n}-1}\right)\right\} \text {; }
\end{aligned}
$$

$(\mathrm{x})$

$$
\begin{aligned}
& d\left(z_{n}, z_{n+1}\right) \leqslant \lambda \cdot d\left(z_{n-2}, z_{n-1}\right)-d\left(z_{n-1}, z_{\mathfrak{n}}\right)+\frac{\lambda^{2}}{q} d\left(z_{n-2}, z_{n-1}\right)+\frac{\lambda}{q} d\left(z_{n}, z_{n+1}\right), \\
& \left(1-\frac{\lambda}{q}\right) d\left(z_{n}, z_{n+1}\right) \leqslant\left(\lambda+\frac{\lambda^{2}}{q}\right) d\left(z_{n-2}, z_{n-1}\right)-d\left(z_{n-1}, z_{n}\right), \\
& \frac{\mathrm{q}-\lambda}{\mathrm{q}} \mathrm{d}\left(z_{\mathrm{n}}, z_{\mathrm{n}+1}\right) \leqslant \frac{\lambda \mathrm{q}+\lambda^{2}}{\mathrm{q}} \mathrm{d}\left(z_{\mathrm{n}-2}, z_{\mathrm{n}-1}\right) \text {, } \\
& d\left(z_{n}, z_{n+1}\right) \leqslant \frac{\lambda q+\lambda^{2}}{q-\lambda} d\left(z_{n-2}, z_{n-1}\right) .
\end{aligned}
$$


From equations (2.4), (2.5), (2.6), (2.7), and (2.8) we obtain

$$
\mathrm{d}\left(z_{\mathfrak{n}}, z_{\mathfrak{n}+1}\right) \leqslant u \max \left\{\mathrm{d}\left(z_{\mathfrak{n}-2}, z_{\mathfrak{n}-1}\right), \mathrm{d}\left(z_{\mathfrak{n}}, z_{\mathfrak{n}-1}\right)\right\},
$$

where

$$
u=\max \left\{\lambda, \lambda+\lambda^{2}, \frac{\lambda}{1-\lambda},\left(\frac{\lambda q+\lambda^{2}}{q-\lambda}\right)\right\}
$$

Combining Cases (1), (2), and (3) we obtain

$$
d\left(z_{n}, z_{n+1}\right) \leqslant u \cdot w_{n},
$$

where $\omega_{\mathfrak{n}} \in\left\{d\left(z_{n-2}, z_{n-1}\right), d\left(z_{n-1}, z_{n}\right)\right\}$ and

$$
u=\max \left\{\lambda, \frac{\lambda}{q-\lambda}, \lambda+\lambda^{2}, \frac{\lambda}{1-\lambda},\left(\frac{\lambda q+\lambda^{2}}{q-\lambda}\right)\right\}=\max \left\{\frac{\lambda}{q-\lambda}, \frac{\lambda}{1-\lambda},\left(\frac{\lambda q+\lambda^{2}}{q-\lambda}\right)\right\} .
$$

Following the procedure of Assad and Kirk [5], it can be easily verified by induction that for $n>1$,

$$
\mathrm{d}\left(z_{\mathrm{n}}, z_{\mathrm{n}+1}\right) \leqslant \mathrm{u}^{\frac{\mathrm{n}-1}{2}} \cdot \omega_{2}
$$

where $\omega_{2} \in\left\{d\left(z_{0}, z_{1}\right), d\left(z_{1}, z_{2}\right)\right\}$.

For $n>m$, using equation (2.9) and the triangle inequality we have

$$
\begin{aligned}
\mathrm{d}\left(z_{\mathfrak{n}}, z_{\mathrm{m}}\right) & \leqslant \mathrm{d}\left(z_{\mathfrak{n}}, z_{\mathfrak{n}-1}\right)+\mathrm{d}\left(z_{\mathfrak{n}-1}, z_{\mathfrak{n}-2}\right)+\cdots+\mathrm{d}\left(z_{\mathrm{m}+1}, z_{\mathrm{m}}\right) \\
& \leqslant\left(u^{\frac{n-1}{2}}+u^{\frac{n-2}{2}}+\cdots+u^{\frac{m-1}{2}}\right) \cdot \omega_{2} \leqslant \frac{\sqrt{u})^{m-1}}{1-\sqrt{u}} \cdot \omega_{2} \rightarrow 0, \quad \text { as } \mathrm{m} \rightarrow \infty .
\end{aligned}
$$

The sequence is Cauchy. Since $z_{n}=f \chi_{n-1} \in f K \cap K$ is complete, it follows that there is some $z \in f K \cap K$ such that $z_{n} \rightarrow z$. Let $w$ in $K$ be such that $T w=z$. By the construction of $\left\{z_{n}\right\}$, there is a subsequence $\left\{z_{n_{k}}\right\}$ such that $z_{n_{k}}=y_{n_{k}}=f x_{n_{k-1}}$ and $f x_{n_{k-1}} \rightarrow z$. We show that $f w=z$,

$$
d(f w, z) \leqslant d\left(f w, f x_{n_{k-1}}\right)+d\left(f x_{n_{k-1}}, z\right) \leqslant \lambda \cdot u_{n_{k}}+d\left(f x_{n_{k-1}}, z\right),
$$

where

$$
\begin{aligned}
u_{n_{k}} \in\{ & \frac{\left(d\left(T w, T x_{n_{k-1}}\right)\right.}{2}, d\left(T x_{n_{k-1}}, f x_{n_{k-1}}\right), d(T w, f w), \min \left\{d\left(T w, f x_{n_{k-1}}\right), d\left(T x_{n_{k-1}}, f w\right)\right\}, \\
& \left.\frac{d\left(T w, f x_{n_{k-1}}\right)+d\left(T x_{n_{k-1}}, f w\right)}{q}\right\} .
\end{aligned}
$$

Taking $z_{n_{k}}=y_{n_{k}}=f x_{n_{k-1}} \rightarrow z$ as $n \rightarrow \infty$ yields

$$
u_{n} \in\left\{0,0, d(z, f w), \min \{0, d(z, f w)\}, \frac{d(z, f w)}{q}\right\}, \quad u_{n} \in\left\{d(z, f w), \frac{d(z, f w)}{q}\right\} .
$$

Thus, we have

(a). $d(f w, z) \leqslant \lambda d(z, f w)+d\left(f x_{n_{k-1}}, z\right) \leqslant \lambda d(z, f w)$.

Since $\lambda<1$, it follows that $\mathrm{d}(\mathrm{f} w, z)=0$. This implies $z=\mathrm{f} w$.

(b). $d(f w, z) \leqslant \frac{\lambda}{q} d(f w, z)$.

Since $\lambda<q$, it follows that $d(f w, z)=0$. Hence $z=f w$.

In all cases we have $z=f w$.

Suppose that $T$ and $f$ are coincidentally commuting. Then, we have $z=f w=T w \Rightarrow f z=f T w=$ $\mathrm{Tf} w=\mathrm{T} z$. Next we prove that $z=\mathrm{f} z=\mathrm{T} z$. Suppose that $z \neq \mathrm{f} z$, then using equation (2.1) we obtain

$$
d(f z, z)=d(f z, f w) \leqslant \lambda \cdot u(z, w),
$$


where

$$
\begin{aligned}
u(z, w) & \in\left\{\frac{d(T z, T w)}{2}, d(T z, f z), d(T w, f w), \min \{d(T z, f w), d(T w, f z)\}, \frac{d(T z, f w)+d(T w, f z)}{q}\right\} \\
& \leqslant\left\{\frac{d(z, z)}{2}, d(z, f z), d(z, z), \min \{d(z, z), d(z, f z)\}, \frac{d(z, z)+d(z, f z)}{q}\right\} \leqslant\left\{d(z, f z), \frac{d(z, f z)}{q}\right\} .
\end{aligned}
$$

Case (1): $d(f z, z) \leqslant \lambda d(f z, z)$. It is a contradiction. Hence $z=f z$.

Case (2): $d(f z, z) \leqslant \frac{\lambda}{q} d(f z, z)$. It is also a contradiction. This implies that $z=f z$. Therefore we obtain $z=f z=T z$. Hence $T$ and $f$ have a common fixed point.

Suppose there exists another common fixed point of $T$ and $f$, say $v$, such that $z=v$. On the other hand, we prove that $z \neq v$. Using equation (2.1), we have

$$
d(z, v)=d(f z, f v) \leqslant \lambda \cdot u(z, v),
$$

where

$$
\begin{aligned}
u(z, v) & \in\left\{\frac{d(T z, T v)}{2}, d(T z, f z), d(T v, f v), \min \{d(T z, f v), d(T v, f z)\}, \frac{d(T z, f v)+d(T v, f z)}{q}\right\} \\
& \leqslant\left\{\frac{d(z, v)}{2}, d(z, z), d(v, v), \min \{d(z, v), d(v, z)\}, \frac{d(z, v)+d(v, z)}{q}\right\} \leqslant\left\{\frac{d(z, v)}{2}, d(z, v), \frac{2}{q} d(z, v)\right\} .
\end{aligned}
$$

Case (1): $d(z, v) \leqslant \frac{\lambda}{2} d(z, v)$. Since $\lambda<1$, it follows that $z=v$.

Case (2): $d(z, v) \leqslant \lambda d(z, v)$. It is a contradiction. Hence $z=v$.

Case (3): $d(z, v) \leqslant \frac{2 \lambda}{q} d(z, v) \leqslant \frac{\lambda}{q} d(z, v)$. In all the cases we have $z=v$. Therefore the common fixed point for $T$ and $f$ is unique.

Remark 2.4. In Theorem 2.2, if $\min \{\mathrm{d}(\mathrm{TX}, \mathrm{Ty}), \mathrm{d}(\mathrm{Ty}, \mathrm{Tx})\}$ is removed from its operator, then we obtain the result of Radenović and Rhoades ([16, Theorem 2.2]) proved in the setting of cone metric spaces. Moreso, Theorem 2.2 generalizes Theorem 4 of Ćirić et al. [9] proved in the setting of Banach spaces without employing the notion of convexity.

Setting $T=I_{x}$, the identity mapping of $X$ in Theorem 2.2, we obtain the following result.

Corollary 2.5. Let $(\mathrm{X}, \mathrm{d})$ be convex metric space, $\mathrm{K}$ a non-empty closed subset of $\mathrm{X}$, and $\delta \mathrm{K}$ the boundary of $\mathrm{K}$. Let $\delta \mathrm{K}$ be nonempty such that for each $\mathrm{x} \in \mathrm{K}$ and $\mathrm{y} \notin \mathrm{K}$ there exists a point $z \in \delta \mathrm{K}$ such that

$$
d(x, z)+d(z, y)=d(x, y)
$$

Suppose that $f: K \rightarrow X$, satisfies the condition

$$
d(f x, f y) \leqslant \lambda \cdot u(x, y),
$$

where

$$
\left.u(x, y) \in\left\{\frac{d(x, y)}{2}, d(x, f x), d(y, f y), \min \{d(x, f y), d(y, f x)\}, \frac{d(x, f y)+d(y, f x)}{q}\right\}\right)
$$

for all $x, y \in K, 0<\lambda<\frac{1}{2}, q>2-\lambda$ and $f$ has the additional property that for each $x \in \delta K$, the boundary of $K, f x \in K$, then $f$ has a unique fixed point.

Example 2.6. Let $X$ be the set of real numbers with the usual metric, $K=[0,+\infty)$ and let $T: K \rightarrow X$ and $f: K \cap T(K) \rightarrow X$ be two non-self mappings defined by $T x=x^{2}$ and $f x=\frac{x^{2}}{4}$ for all $x \in K$.

Thus $T$ and $f$ satisfied equation (2.1) and all the hypotheses in Theorem 2.2 are satisfied. So, $T$ and $f$ have a unique common fixed point $z=0$. 
To see that our result generalized Theorem 2.2 of Radenović and Rhoades [16], let $\lambda=\frac{1}{2}, x=0$, and $y=1$, then we have

$$
d(f x, f y) \leqslant \frac{1}{2}\left(\left|\frac{0}{4}-\frac{1}{4}\right|\right)=\frac{1}{2}\left(\left|0-\frac{1}{4}\right|\right)=\frac{1}{2} d(T 0, f 1) \leqslant \lambda d(T x, f y) .
$$

This does not satisfy Theorem 2.2 of Radenović and Rhoades [16].

Conclusion 2.7. This research introduced a new class of nonlinear contractive non-self mappings in metric spaces. Furthermore, the existence and uniqueness of common fixed point for a pair of these maps is proved in the setting of convex metric space. The obtained result is more general than the result of Radenović and Rhoades [16] and Ćirić et al. [9] in the setting of cone metric spaces of Haung and Zang [10] and Banach spaces, respectively. Example is given to support our claim.

\section{References}

[1] M. Abbas, J. Jungck, Common fixed point results for noncommuting mappings without continuity in cone metric spaces, J. Math. Anal. Appl., 341 (2008), 416-420. 1.1

[2] T. Abdeljawad, E. Karapınar, K. Taş, Existence and uniqueness of a common fixed point on partial metric spaces, Appl. Math. Lett., 24 (2011), 1900-1904. 1

[3] H. Akewe, G. A. Okeke, Convergence and stability theorems for the Picard-Mann hybrid iterative scheme for a general class of contractive -like operators, J. Fixed Point Theory Appl., 2015 (2015), 8 pages. 1

[4] N. A. Assad, On a fixed point theorem in Banach space, Tamkang J. Math., 7 (1976), 91-94. 1

[5] N. A. Assad, W. A. Kirk, Fixed point theorems for set-valued mappings of contractive type, Pacific J. Math., 43 (1972), 553-562. 1, 2

[6] H. Aydi, A. Felhi, E. Karapınar, On common best proximity points for generalized alpha-psi-proximal contraction, J. Nonlinear Sci. Appl., 9 (2016), 2658-2670. 1

[7] H. Aydi, M. Jellahi, E. Karapinar, Common fixed points for generalized $\alpha$-implicit contractions in partial metric spaces: consequences and application, Rev. R. Acad. Cienc. Exactas Fs. Nat. Ser. A Math. RACSAM, 109 (2015), 367-384. 1

[8] L. B. Ćirić, Contractive-type non-self mappings on metric spaces of hyperbolic type, J. Math. Anal. Appl., 317 (2006), 28-42. 1

[9] L. Ćirić, V. Rakočević, S. Radenović, M. Rajović, R. Lazović, Common fixed point theorems for non-self mappings in metric spaces of hyperbolic type, J. Comput. Appl. Math., 233 (2010), 2966-2974. 1, 1, 2.4, 2.7

[10] L.-G. Huang, X. Zhang, Cone metric spaces and fixed point theorems of contractive mappings, J. Math. Anal. Appl., 332 (2007), 1468-1476. 1, 2.7

[11] M. Imdad, S. Kumar, Rhoades-type fixed-point theorems for a pair of nonself mappings, Comput. Math. Appl., 46 (2003), 919-927. 1

[12] E. Karapinar, A note on common fixed point theorem in partial metric spaces, Miskolic Math. Notes, 12 (2011), $185-191$. 1

[13] W. A. Kirk, Krasnoselskii's iteration process in hyperbolic space, Numer. Funct. Anal. Optim., 4 (1982), 371-381. 1

[14] G. A. Okeke, M. Abbas, Convergence and almost sure T-stability for a random iterative sequence generated by a generalized random operator, J. Inequal. Appl., 2015 (2015), 11 pages. 1

[15] G. A. Okeke, J. K. Kim, Convergence and summable almost T-stability of the random Picard-Mann hybrid iterative process, J. Inequal. Appl., 2015 (2015), 14 pages. 1

[16] S. Radenović, B. E. Rhoades, Fixed point theorem for two non-self mappings in cone metric spaces, Comput. Math. Appl., 57 (2009), 1701-1707. 1, 2.4, 2.6, 2.7

[17] B. E. Rhoades, A fixed point theorem for some non-self mappings, Math. Japon., 23 (1978), 457-459. 1

[18] R. Sumitra, V. R. Uthariaraj, R. Hemavathy, P. Vijayaraju, Common fixed point theorem for non-self mappings satisfying generalized Ciric type contraction condition in cone metric space, J. Fixed Point Theory Appl., 2010 (2010), 17 pages. 1

[19] W. Takahashi, A convexity in metric spaces and nonexpansive mappings, I, Kodai Math. Sem. Rep., 22 (1970), 142-149. 1 